\title{
Steamed Ginger Extract Exerts Anti-inflammatory Effects in Helicobacter pylori-infected Gastric Epithelial Cells through Inhibition of NF-kB
}

\author{
Moon-Young Song', Da-Young Lee', Sang-Yong Park², Seul-A Seo², Jeong-Seung Hwang², Soo-Hyeon Heo², \\ Eun-Hee Kim ${ }^{1}$ \\ ${ }^{1}$ Institute of Pharmaceutical Sciences, College of Pharmacy, CHA University, Seongnam, ${ }^{2}$ SD Biotechnologies Co., Ltd., \\ Seoul, Korea
}

\begin{abstract}
Ginger (Zingiber officinale) has traditionally been used as a treatment for inflammatory diseases in the Asian region. Recently, anti-inflammatory effects of steamed ginger extract (GGE03) have been reported, but its association with Helicobacter pylori $(H$. pylori)-induced gastritis has not been investigated. The purpose of this study was to assess the anti-inflammatory activity of GGE03 in $H$. pylori-infected gastric epithelial cells. Our studies revealed that the GGE03 suppressed the growth of $H$. pylori. GGE03 markedly reduced the expression of the $H$. pylori-induced pro-inflammatory cytokines including interleukin (IL)-8, TNF- $\alpha$, IL-6, inducible NOS (iNOS) and IFN- $\gamma$. We also demonstrated that GGE03 treatment inhibited the $H$. pylori-activated NF-kB signaling pathway. In addition, the treatment with GGE03 significantly attenuated nitric oxide production and myeloperoxidase activity in $\mathrm{H}$. pylori-infected gastric epithelial cells. These anti-inflammatory effects of GGE03 were more effective than ginger extract. Finally, we investigated the minimum effective concentration of GGE03 to inhibit $H$. pylori-induced inflammation. Our findings suggest that GGE03 not only inhibits the growth of $H$. pylori, but also attenuates $H$. pylori-induced inflammation.
\end{abstract}

Key Words Steamed ginger extract, Helicobacter pylori, Gastritis, Anti-inflammation, NF-kappa B

\section{INTRODUCTION}

Ginger (Zingiber officianale) is one of the herbaceous perennial plants that belongs to the Zingiberaceae family. Ginger has been used to treat stomachache, fever, vomiting and primary dysmenorrhea [1,2], and has an anti-inflammatory, anti-microbial and anti-oxidative properties [3]. Interestingly, recent studies have revealed that steamed ginger has a lower toxicity and higher efficacy than the dried ginger $[4,5]$. During the process of steaming, the bioactive components were affected, and this apparently enhanced the biological activities of ginger [6]. Actually, steamed ginger has been shown to have a greater anti-hyperglycemic, anti-diabetic and anti-obesity activity [6].

Helicobacter pylori (H. pylori) infection is a major risk factor for gastric diseases, including chronic gastritis and gastric adenocarcinoma [7]. It is estimated that more than half of the world's population is infected with $H$. pylori, and the
WHO classified this bacterium as a class 1 carcinogen [8]. Infection by $\mathrm{H}$. pylori activates several pro-inflammatory signals. NF-kB is one of the major pro-inflammatory pathways activated during infection by $H$. pylori [9]. The inflammatory response induced by $H$. pylori infection is implicated in the pathogenesis of some gastric disorders. Therefore, inhibition of inflammatory response is important in the management of gastric disease associated with $H$. pylori infection [10]. It is well known that NF-kB activation by $H$. pylori infection is mediated through cytotoxin-associated gene A (CagA), lipopolysaccharide, and peptidoglycan [11]. In the present study, we investigated the inhibitory effect of steamed ginger extract (GGE03) against the expression of inflammatory cytokines, and production of cellular nitric oxide (NO), and myeloperoxidase (MPO) activity in AGS human gastric adenocarcinoma cells infected with $H$. pylori. 


\section{MATERIALS AND METHODS}

Preparation of steamed ginger extract (GGE03) GGE03 was prepared as previously published [5]. Briefly, ginger was washed three times with distilled water, dried at $50^{\circ} \mathrm{C}$ for 30 hours, and then steamed at: 2 to $2.5 \mathrm{kgf} / \mathrm{cm}^{2}, 97^{\circ} \mathrm{C}$, for 2 hours. GGE03 was obtained by extracting steamed ginger with fifteen-fold $70 \%$ ethanol $(\mathrm{v} / \mathrm{v})$ for 15 hours at $85^{\circ} \mathrm{C}, 1.5$ $\mathrm{kg} / \mathrm{cm}^{2}$, and then passed through a 60-mesh filter, concentrated at $-650 \mathrm{mmHg}, 55^{\circ} \mathrm{C}$. GE was prepared in the same way without steam process. The extract (GE and GGE03) was spray-dried to obtain a powder and stored at $-20^{\circ} \mathrm{C}$ until use. GE and GGE03 were dissolved in distilled water and diluted with medium into appropriate concentrations.

\section{Cell culture}

AGS gastric adenocarcinoma cells were purchased from the American Type Culture Collection (ATCC, Manassas, VA, USA) and maintained according to the ATCC's instructions. Cells were cultured in Roswell Park Memorial Institute (RPMI) 1640 Medium (GE Healthcare, Chicago, IL, USA) supplemented with $10 \%$ (v/v) fetal bovine serum (ATCC), $100 \mathrm{U} / \mathrm{mL}$ penicillin and $100 \mu \mathrm{g} / \mathrm{mL}$ streptomycin. Cells were incubated at $37^{\circ} \mathrm{C}$ in a humidified atmosphere containing $5 \% \mathrm{CO}_{2}$.

\section{Bacterial culture and infection of gastric cells} The H. pylori strain ATCC 43504 (American Type Culture Collection, a cagA+ and vacA s1-m1 type's strain) was obtained from ATCC. H. pylori were cultured at $37^{\circ} \mathrm{C}$ in BBL Trypticase soy (TS) agar plate with $5 \%$ sheep blood (TSAll; BD Biosciences, Franklin Lakes, NJ, USA) under microaerophilic condition (BD GasPaK EZ Gas Generating Systems; $\mathrm{BD}$ Biosciences) for 3 days. The bacteria were harvested in clean TS broth, centrifuged at $3,000 \times g$ for 5 minutes, and resuspended in broth at a final density of $10^{9} \mathrm{CFUs} / \mathrm{mL}$. For infection, an absorbance of 0.4 units at $600 \mathrm{~nm}$ was considered equivalent to $1.4 \times 10^{6}$ bacteria. Cells were infected with a multiplicity of infection (MOI) of 1:50.

\section{Cytotoxicity assay}

For measuring the viability of AGS cells, the cells were treated with GE and GGE03 at the concentrations of 1, 10, 100, or $200 \mu \mathrm{g} / \mathrm{mL}$. After incubation for 24 hours, cells were rinsed with PBS and then MTT (Sigma-Aldrich, St. Louis, MO, USA) solution was added to the cells followed by incubation for additional 3 hours. After the conversion of the substrate to a chromogenic product by metabolically active cells, the medium was removed and the purple MTT formazan crystals were solubilized with dimethyl sulfoxide (DMSO). The absorbance of each samples measured at $570 \mathrm{~nm}$ as a reference wavelength.

\section{Western blotting}

This assay was performed as previously described [12]. Brief- ly, proteins were separated by SDS PAGE and transferred to a nitrocellulose membrane. The membrane was incubated with optimal concentrations of a primary antibody (pp65, p65, pp50, p50, p-IкB $\alpha, I_{\kappa} B \alpha$ or $\beta$-actin) at $4^{\circ} \mathrm{C}$ overnight and then incubated with an appropriate secondary antibody for 1 hour at room temperature. The immune-labeled proteins were visualized using an enhanced chemiluminescence system (Thermo Fisher Scientific, Waltham, MA, USA). The primary antibodies to detect pp65, p65, pp50, p50, p- $I_{\kappa} B \alpha$, and $I_{\kappa} B \alpha$ used in this study were purchased from Cell Signaling Technology (Danvers, MA, USA) and $\beta$-actin from Santa Cruz Biotechnology (Dallas, TX, USA).

\section{RNA preparation and gene expression analysis} AGS cells were washed twice with PBS, and total mRNA was isolated from the cells using TRIzol ${ }^{\mathrm{TM}}$ Reagent (Invitrogen, Waltham, MA, USA), and CDNA was prepared using a Labopass cDNA synthesis kit (Cosmogenetech, Seoul, Korea) according to the manufacturer's instructions. The mRNA levels were assessed by reverse transcription PCR (RT-PCR) and quantitative real-time PCR (qRT-PCR). qRT-PCR was performed as previously and assessed on the $\mathrm{ViiA}^{\mathrm{TM}} 7$ real-time PCR system (Applied Biosystems, Waltham, MA, USA) using Luna universal qPCR master mix (New England Biolabs, Beverly, MA, USA). The relative quantities of target genes were calculated from triplicate samples after normalization by an internal control, 18S rRNA. The PCR primer sequences used in this study are listed in Table 1.

\section{Myeloperoxidase activity assay}

Samples obtained from cellular or bacterial pellets were washed twice with PBS and then lysed with a buffer containing $20 \mathrm{mM}$ HEPES. Then, measurement of MPO activity was performed with kits (Abcam, Cambridge, UK) according to the manufacturer's instructions. The activity was measured as the absorbance at $450 \mathrm{~nm}$ read by a 96 -well microplate reader.

\section{Measurement of NO production}

Culture medium supernatants were collected from the culture of AGS cells. The NO production in the culture medium was quantified colorimetrically using a nitric oxide detection kit (iNtRON Biotechnology, Seongnam, Korea). The absorbance at $550 \mathrm{~nm}$ was read with a 96-well microplate reader.

\section{Disk diffusion assay}

H. pylori $\left(1.4 \times 10^{6} \mathrm{CFU} / \mathrm{mL}\right)$ suspended in $100 \mu \mathrm{L}$ of PBS were applied onto the $5 \%$ Sheep Blood Agar (BD Bioscience) plate and spread on it. Then, 6-mm paper disks impregnated with different concentrations of GE and GGE03 (10 and 100 $\mathrm{mg} / \mathrm{mL}$ ) were positioned on an agar plate to evaluate their anti-bacterial effect. After incubation at $37^{\circ} \mathrm{C}$ in a microanaerobic chamber for 72 hours, the diameters of the growth inhibition zone were measured. 
Table 1. Primer sequences used in RT-PCR and qRT-PCR primers

\begin{tabular}{|c|c|c|c|}
\hline Species & Gene & & Primer sequence \\
\hline \multirow[t]{4}{*}{ Human (RT-PCR) } & $18 S$ rRNA & Foward & CCCAACTTCTTAGAGGGACAAGT \\
\hline & & Reverse & TAGTCAAGTTCGACCGTCTTCTC \\
\hline & iNOS & Foward & GGCCTCTCAGCTCACCCCGA \\
\hline & & Reverse & CCAGGCGCACTGTCTGGTGG \\
\hline \multirow[t]{14}{*}{ Human (qRT-PCR) } & $18 S$ rRNA & Foward & GCAATTATTCCCCATGAACG \\
\hline & & Reverse & GGCCTCACTAAACCATCCAA \\
\hline & IL-8 & Foward & TCC TTG TTC CAC TGT GCC TTG \\
\hline & & Reverse & TGC TTC CAC ATG TCC TCA CAA \\
\hline & $T N F-\alpha$ & Foward & TCA GAG GGC CTG TAC CTC AT \\
\hline & & Reverse & GGA AGA CCC CTC CCA GAT AG \\
\hline & $I L-6$ & Foward & AGGGCTCTTCGGCAAATGTA \\
\hline & & Reverse & GAAGGAATGCCCATTAACAACAA \\
\hline & $I L-1 \beta$ & Foward & TTA AAG CCC GCC TGA CAG A \\
\hline & & Reverse & GCG AAT GAC AGA GGG TTT CTT \\
\hline & iNOS & Foward & AGGTCCAAATCTTGCCTGGG \\
\hline & & Reverse & ATCTGGAGGGGTAGGCTTGT \\
\hline & $I F N-\gamma$ & Foward & ACTGTCGCCAGCAGCTAAAA \\
\hline & & Reverse & TATTGCAGGCAGGACAACCA \\
\hline
\end{tabular}

RT-PCR, reverse transcription PCR; qRT-PCR, quantitative real-time PCR; iNOS, inducible NOS; IL, interleukin.

A

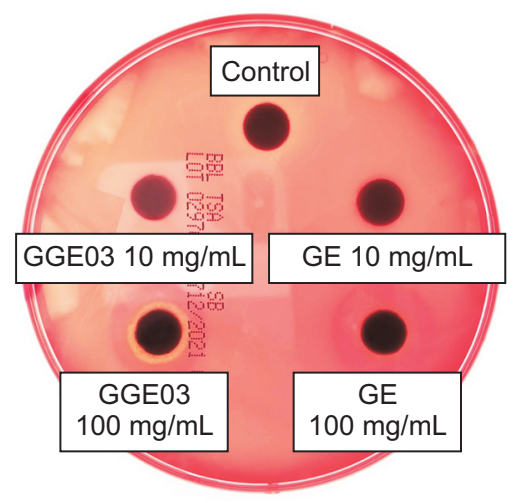

H. pylori $\left(1.4 \times 10^{6} \mathrm{CFU}\right)$

B

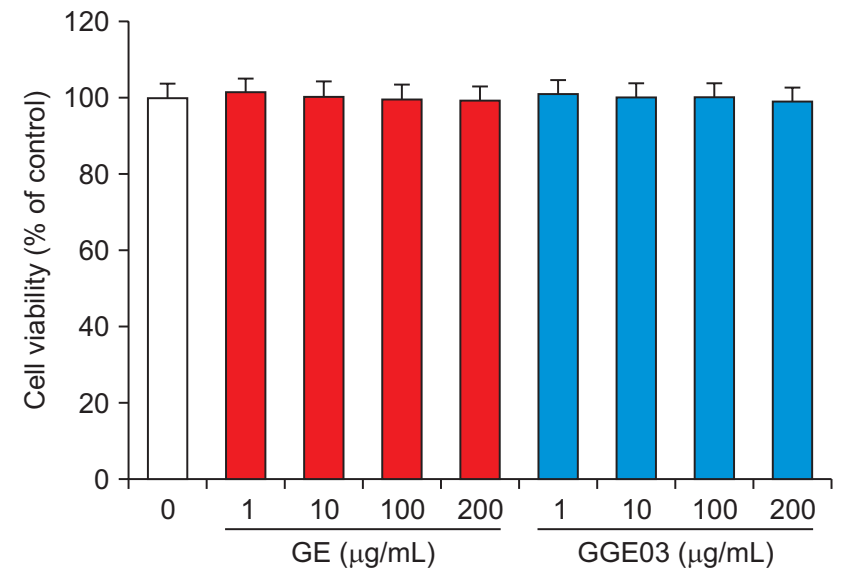

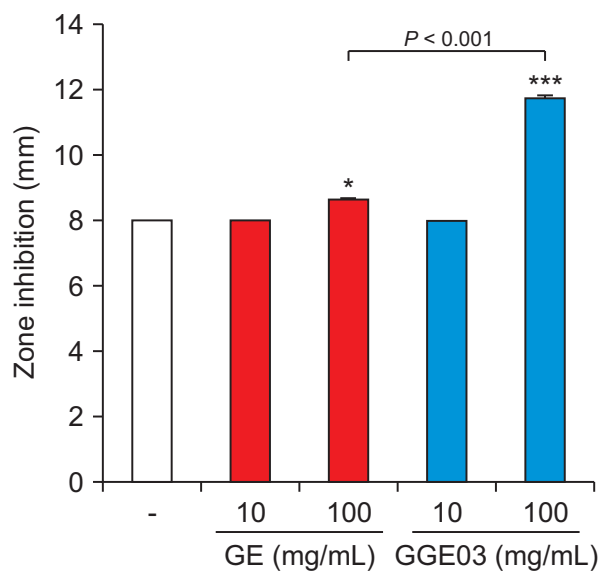

Figure 1. The steamed ginger extract (GGE03) inhibits the growth of Helicobacter pylori (H. pylori) and has no cytotoxic effect in AGS cells. (A) H. pylori strains (ATCC 43504; ATCC, Manassas, VA, USA) were grown on tryptic soy agar plate with $5 \%$ sheep blood containing indicated concentrations of GE or GGE03 (10 and $100 \mathrm{mg} / \mathrm{mL}$ ). H. pylori with GE and GGE03 were determined after 72 hours of incubation. The diameters were calculated against inhibition zone. The results were evaluated by ANOVA. Data are the mean $\pm S D(n=$ 3). Statistical significance was analyzed by ANOVA. ${ }^{*} P<0.05$ and ${ }^{* * *} P<0.001$ compared to control. (B) AGS cells were treated with GE and GGE03 at indicated concentrations for 24 hours and cell viability was measured by the MTT assay. 


\section{Statistical analysis}

Results are expressed as the mean \pm SD. The statistical significance was analyzed by one-way ANOVA. Statistical significance was accepted at $P<0.05$.

\section{RESULTS}

Inhibitory effect of GGE03 on the growth of $\boldsymbol{H}$. pylori in AGS cells

We conducted the investigation of relative inhibitory potency of GGE03 and GE against $H$. pylori growth, using the disk agar diffusion assay. To quantify the inhibitory effect of $H$. pylori, the diameter of growth inhibition area was measured and expressed in millimeters. GGE03 and GE were tested at concentrations of 10 and $100 \mathrm{mg} / \mathrm{mL}$. As shown in Figure 1A, GGE03 at $100 \mathrm{mg} / \mathrm{mL}$ showed an inhibitory effect on $H$. pylori growth with inhibition zone ranging up to $12 \mathrm{~mm}$. However, a lower concentration of GGE03 and GE $(10 \mathrm{mg} / \mathrm{mL})$ was not inhibitory. Next, we investigated the cytotoxic effect of GGE03 and GE on AGS cells. The AGS cells were treated with GGE03 and GE at various concentrations (1, 10, 100, or $200 \mu \mathrm{g} / \mathrm{mL}$ ) for 24 hours. The results showed that GGE03 and GE did not exhibit cytotoxic effects when treated to AGS cells for 24 hours (Fig. 1B).

\section{Anti-inflammatory effect of GGE03 in $\mathbf{H}$. pylori- infected AGS cells}

It has been reported that $H$. pylori infection stimulates the production of pro-inflammatory cytokines such as interleukin (IL)8 , TNF- $\alpha$, IL-6 and IL-1 $\beta$ [13]. The qRT-PCR results showed that the levels of aforementioned cytokines were significantly increased in $\mathrm{H}$. pylori-infected AGS cells, and this was signifi-
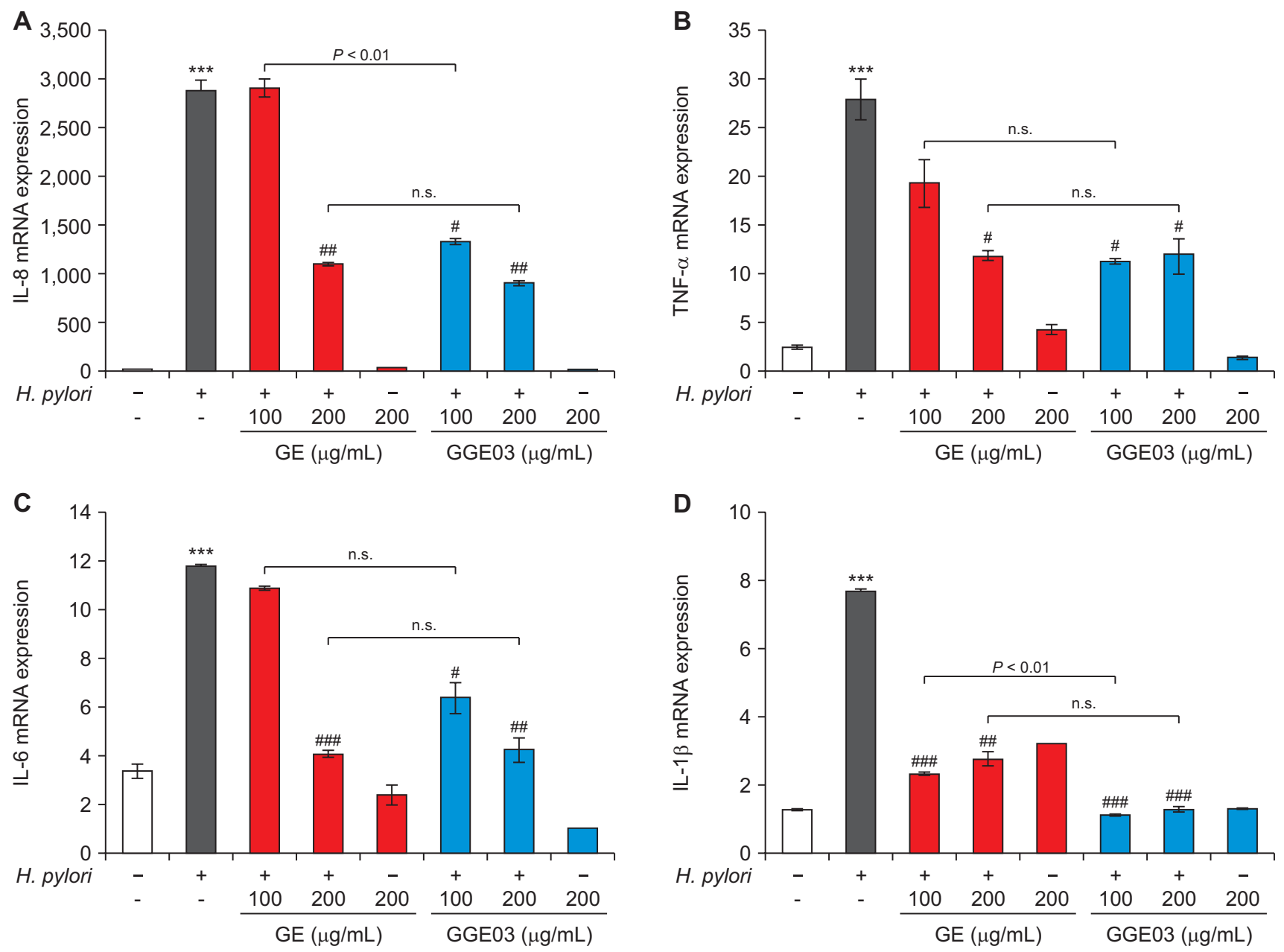

Figure 2. GGE03 inhibits pro-inflammatory cytokine gene expression in H. pylori-infected AGS cells. AGS cells were pretreated with GE and GGE03 at indicated concentrations for 1 hour, followed by co-culture with $H$. pylori (50 multiplicity of infection) for additional 3.5 hours. The effects of GE and GGE03 on the mRNA expression of IL-8 (A), TNF- $\alpha$ (B), IL-6 (C), and IL-1 $\beta$ (D) in AGS cells were analyzed by quantitative real-time PCR (qRT-PCR). 18S rRNA was used as an internal control for the expression of pro-inflammatory cytokines. Data are the mean \pm SD. Statistical significance was analyzed by ANOVA. IL, interleukin; H. pylori, Helicobacter pylori; n.s., not significant. ${ }^{* \star *} P<0.001$ compared to control; ${ }^{\sharp} P<0.05$, ${ }^{\#} P<0.01$, and ${ }^{\# \#} P<0.001$ compared to the $H$. pylori group. 
cantly reduced by GGE03 and GE treatment (Fig. 2). GGE03 was shown to more effectively inhibit $H$. pylori-induced inflammation than GE in AGS cells.

\section{GGE03 exerts anti-inflammatory effects through blockade of $\boldsymbol{H}$. pylori-induced NF-KB signaling pathway in AGS cells \\ The NF- $\mathrm{NB}$ signaling pathway is one of the most important} mediators of pro-inflammatory cytokine production upon $\mathrm{H}$. pylori infection $[14,15]$. As shown in Figure $3 A$, the phosphorylation of $I_{\kappa} B \alpha$ was enhanced by $H$. pylori infection, and GGE03 and GE treatment significantly reduced the levels of phosphorylated $I_{\kappa} \mathrm{B} \alpha$. GGE03 was more effective in inhibiting the phosphorylation of $I_{\kappa} \mathrm{B} \alpha$ induced by $H$. pylori than GE. Moreover, phosphorylation of p65, a major functionally active subunit of NF-kB, was increased in $H$. pylori-infected AGS cells, which was significantly inhibited by the treatment with GGE03. In contrast, there was no change in the phosphorylation of $\mathrm{p} 50$ by infection with $H$. pylori or treatment with GGE03 (Fig. 3B). Collectively, these results suggest that GGE03 suppresses $H$. pylori-induced inflammatory response by inhibiting the NF-kB signaling pathway.

\section{GGE03 inhibits the production of NO in $H$. pylori-infected AGS cells}

Several lines of evidence indicate that $\mathrm{NO}$ is involved in the pathogenesis of $H$. pylori-mediated gastritis and gastric cancer. NO has the ability to diffuse freely across the cells and has strong reactivity as a free radical molecule [16]. Therefore, we measured the expression of inducible NOS (iNOS), a prototype pro-inflammatory enzyme responsible for NO production. The results showed that GGE03 treatment significantly decreased the mRNA expression of iNOS as determined by RT-PCR and qRT-PCR in H. pylori-infected AGS cells in a dose-dependent manner (Fig. 4A). GGE03 also inhibited the iNOS-induced production of NO in AGS cells to a greater extent than GE (Fig. 4B).

\section{Effect of GGE03 on H. pylori-induced MPO activity and production of IFN $-\gamma$ in AGS cells} MPO activity is used as an indicator of neutrophil infiltration in inflamed site [17]. Because of the abundance of this enzyme, it is used as an index for $H$. pylori-induced infiltration of neutrophils [18]. As shown in Figure 5, MPO activity was significantly increased in $\mathrm{H}$. pylori-infected AGS cells compared to that in non-treated cells. GGE03 treatment attenuated the
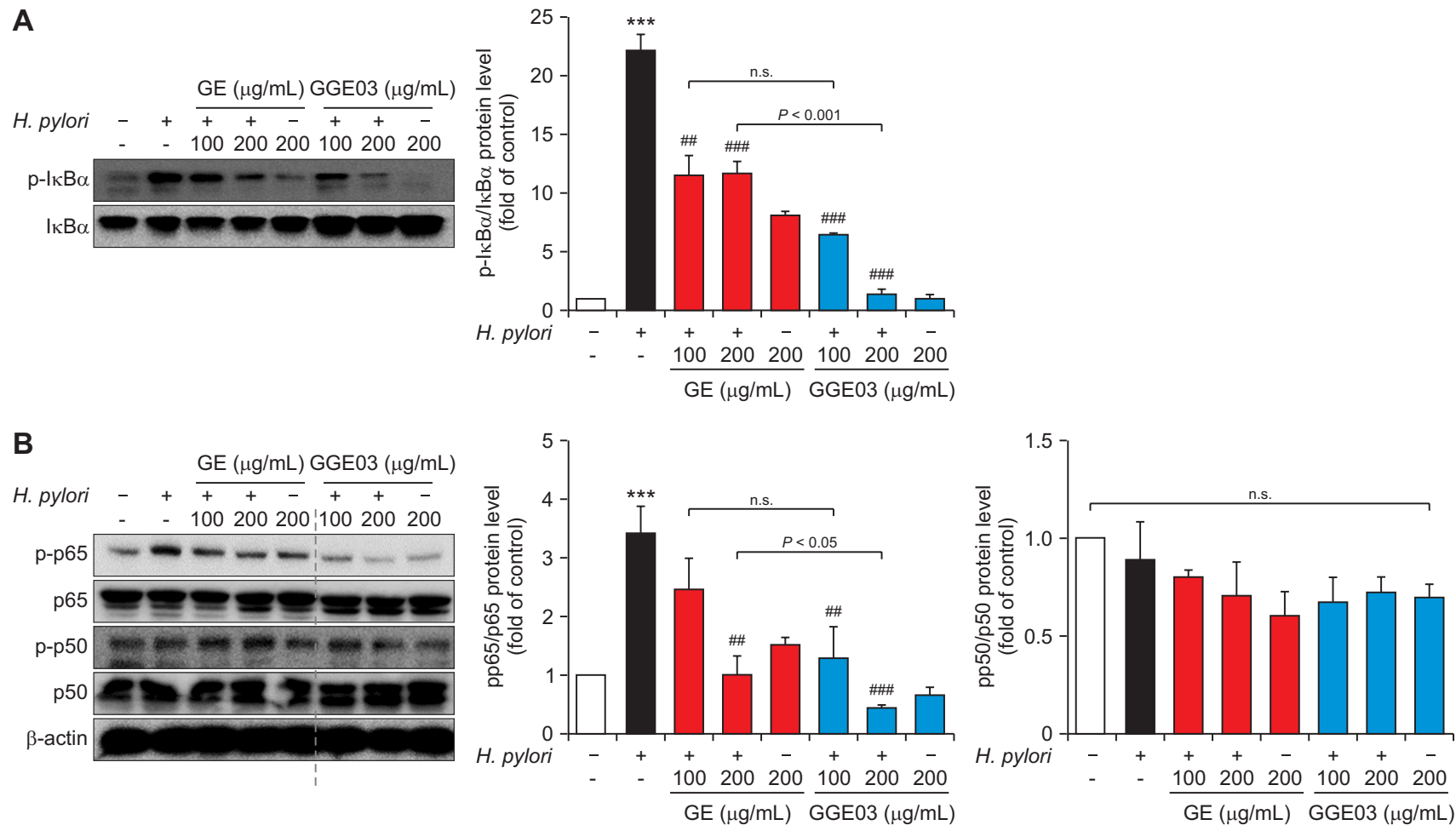

Figure 3. GGE03 exerts anti-inflammatory effects by inhibiting the NF-KB signaling pathway in Helicobacter pylori $(H$. pylori)-infected AGS cells. (A) AGS cells were pretreated with indicated concentrations of GE and GGE03 for 1 hour and then infection was induced by $H$. pylori (50 multiplicity of infection [MOI]) for 1 hour. The expression of $p-I_{\kappa} B \alpha$ and $I_{\kappa} B \alpha$ in AGS cells were determined by Western blotting. (B) AGS cells were pretreated with indicated concentrations of GE and GGE03 for 1 hour and then infection was induced by $H$. pylori (50 MOI) for 1 hour. The expression of p-p65, p65, p-p50, p50 and $\beta$-actin in AGS cells was determined by Western blotting. Results are presented as the mean \pm SD. Statistical significance was analyzed by ANOVA. n.s., not significant. ${ }^{* \star *} P<0.001$ compared to control; ${ }^{\#} P<0.01$ and ${ }^{\# \#} P<0.001$ compared to the $H$. pylori group. 

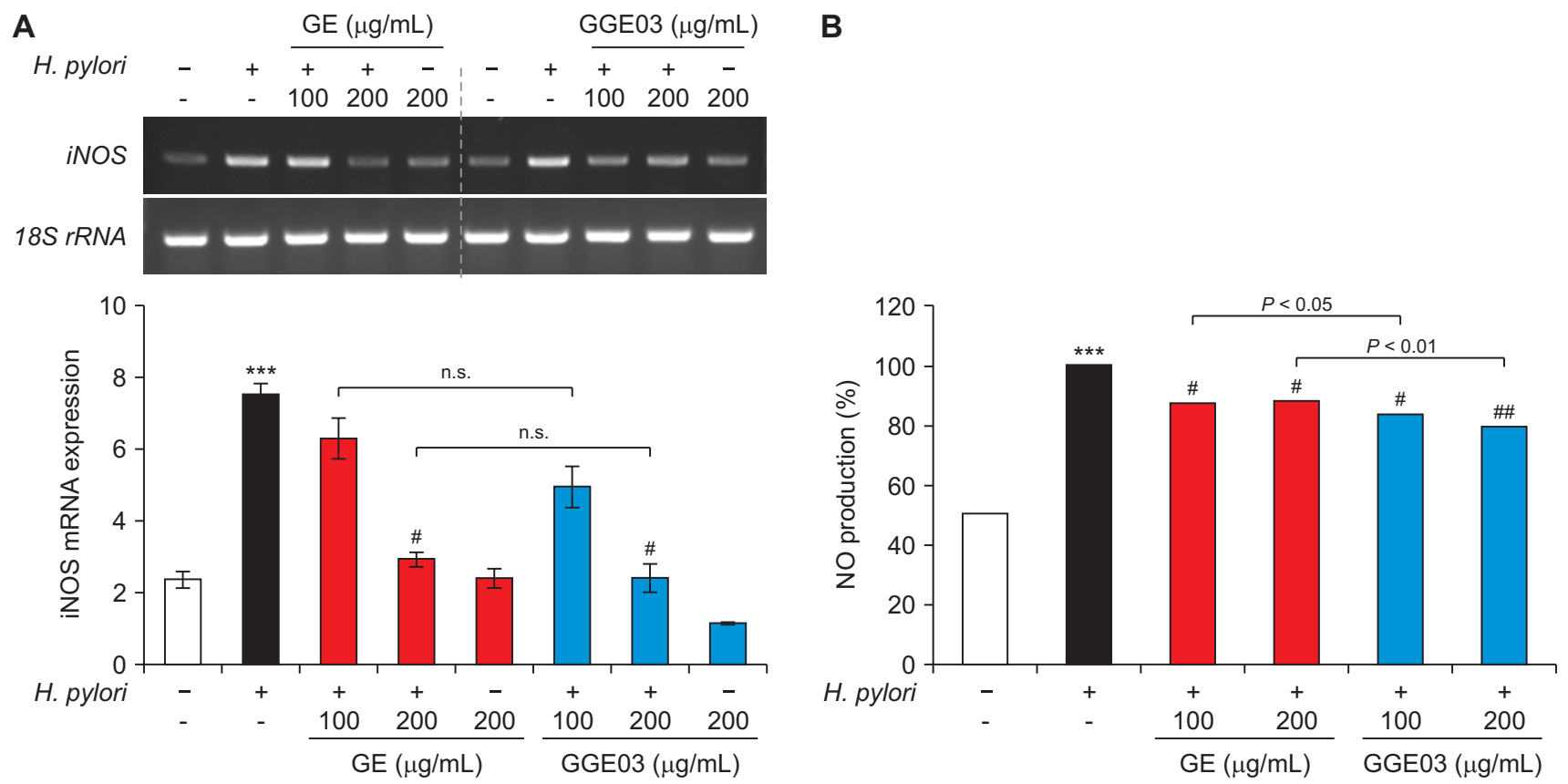

Figure 4. GGE03 inhibits the production of nitric oxide (NO) through inducible NOS (iNOS) in Helicobacter pylori (H. pylori)-infected AGS cells. (A) AGS cells were pretreated with GE and GGE03 indicated concentrations for 1 hour, followed by 1.5 hours co-culture with $\mathrm{H}$. pylori (50 multiplicity of infection [MOI]). After incubation, the culture supernatants were collected for measurement of NO. (B) AGS cells were pretreated with GE and GGE03 at indicated concentrations for 1 hour, followed by 3.5 hours co-culture with $\mathrm{H}$. pylori (50 MOI). The effect of GE and GGE03 on the expression of iNOS in AGS cells was analyzed by reverse transcription PCR (RT-PCR) and quantitative real-time PCR (qRT-PCR). 18S rRNA was used as an internal control for the expression of cytokines. Data are the mean \pm SD. Statistical significance was analyzed by ANOVA. n.S., not significant. ${ }^{* * *} P<0.001$ compared to control; ${ }^{\#} P<0.05,{ }^{\# \#} P<0.01$, and ${ }^{\# \#} P<0.001$ compared to the $H$. pylori group.
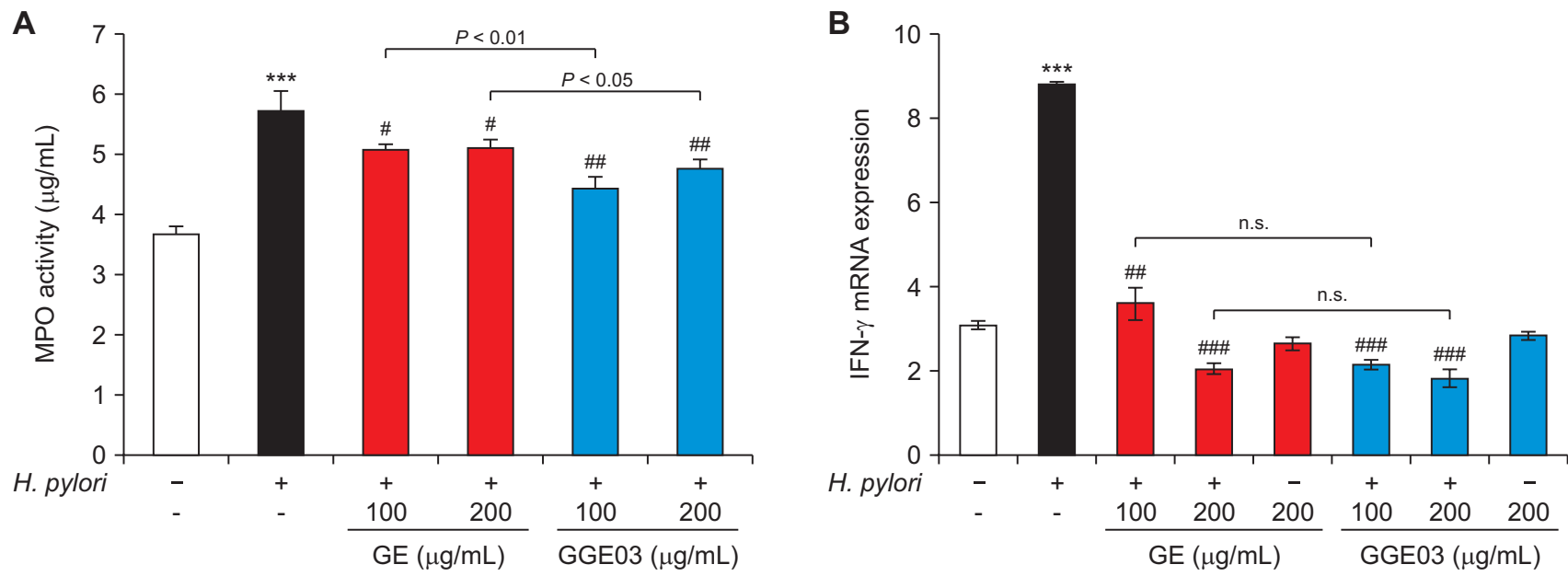

Figure 5. GGE03 inhibits Helicobacter pylori (H. pylori)-induced myeloperoxidase (MPO) activity and expression of IFN-y in AGS cells. (A) AGS cells were pretreated with GE and GGE03 at indicated concentrations for 1 hour, followed by 1.5 hours co-culture with $H$. pylori (50 multiplicity of infection [MOI]). After incubation, the cell lysates were collected for determination of MPO activity as described in Materials and Methods. (B) AGS cells were pretreated with GE and GGE03 indicated concentrations for 1 hour, followed by 12 hours co-culture with $\mathrm{H}$. pylori (50 MOI). The effect of GE and GGE03 on the expression of IFN- $\gamma$ in AGS cells was analyzed by qRT-PCR. 18S rRNA was used as an internal control. Data are the mean \pm SD. Statistical significance was analyzed by ANOVA. n.s., not significant. ${ }^{* * *} P<0.001$ compared to control; ${ }^{\#} P<0.05$, ${ }^{\#} P<0.01$, and ${ }^{\# \#} P<0.001$ compared to the $H$. pylori group.

$H$. pylori-induced elevation of MPO activity in AGS cells (Fig. $5 A$ ). Increased expression of IFN- $\gamma$ after $H$. pylori infection has been reported in the gastric tissues of humans [19]. $H$. pylori is known to stimulate IFN- $\gamma$ production from peripheral blood mononuclear cells and lymphocytes [20]. H. pylori infection significantly increased the mRNA levels of IFN- $\gamma$, and the treatment with GGE03 lowered IFN- $\gamma$ levels in a dose dependent manner (Fig. 5B). These results demonstrate that 
GGE03 may inhibit $H$. pylori-induced neutrophil influx and the expression of inflammatory mediators in AGS cells.

\section{The minimum inhibitory concentration for the anti-inflammatory effect of GGEO3 in $\mathrm{H}$. pylori- infected AGS cells}

To assess the minimum concentration of GGE03 exerting anti-inflammatory responses in $\mathrm{H}$. pylori-infected AGS cells, we monitored the expression of the pro-inflammatory cytokines upon treatment with GGE03 at the concentrations of 50, 100, and $200 \mu \mathrm{g} / \mathrm{mL}$. As qRT-PCR data shown in Figure 6, GGE03 differentially inhibits the mRNA levels of IL-8, TNF- $\alpha$, IL-6 and iNOS at the concentration of $50 \mu \mathrm{g} / \mathrm{mL}$ or higher in $\mathrm{H}$. pylori-infected AGS cells. Thus, $50 \mu \mathrm{g} / \mathrm{mL}$ of GGE03 significantly inhibited the expression of IL-8 mRNA, a major pro-inflammatory cytokine expressed upon $\mathrm{H}$. pylori infection in AGS cells whereas TNF- $\alpha$, IL-6 and iNOS were not altered (Fig. 6).

\section{DISCUSSION}

$H$. pylori infection occurs more than $50 \%$ of the world's population and is widely known as the cause of gastric diseases [21]. H. pylori infection promotes gastritis, gastric ulcer, chronic gastritis, and gastric cancer by changing the stomach $\mathrm{pH}$ [8]. When H. pylori is attached to gastric epithelial cells, it releases various virulence factors such as CagA and vacuolating cytotoxin (VacA) into the epithelial cells using a type IV secretion system [22]. In particular, CagA acts as a signaling molecule in the cell and induces upregulation of $\mathrm{NF}-\kappa \mathrm{B}$, extracellular signal-regulated kinase and IL-8 [23]. Chronic inflammation caused by $\mathrm{H}$. pylori can lead to the development of gastric adenocarcinoma [24]. However, there are limitations for treatment for $H$. pylori infection due to antibiotic resistance $[25,26]$. Therefore, development of new agents to prevent or treat gastric damage caused by $H$. pylori is still needed.

It has been reported that $H$. pylori has different virulence factors released by each strain [27]. There are several differ-

B

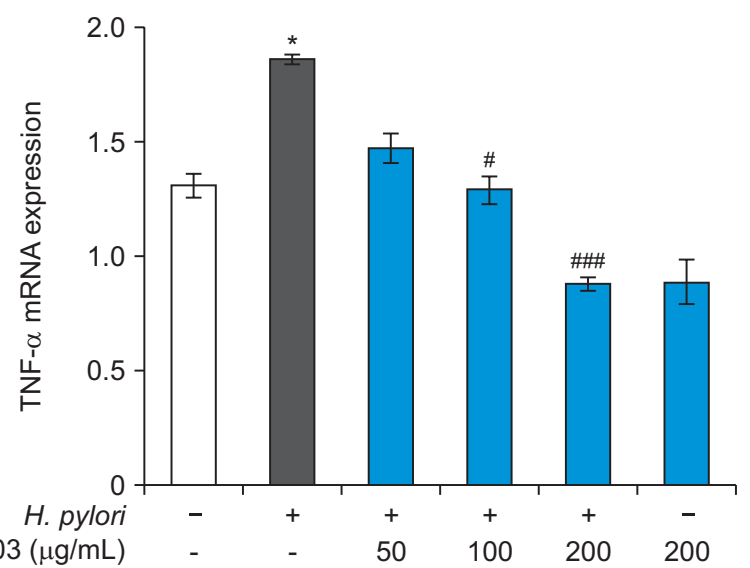

D

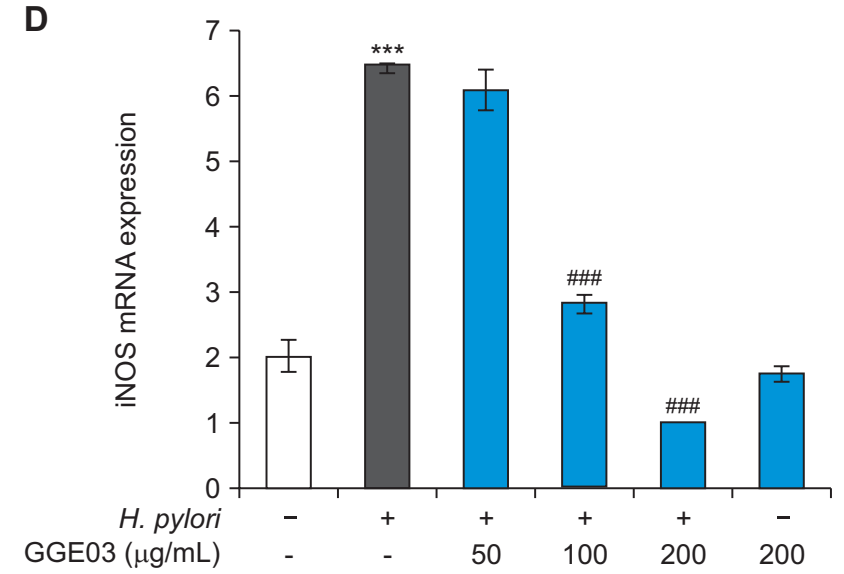
GGE03 $(\mu \mathrm{g} / \mathrm{mL})$

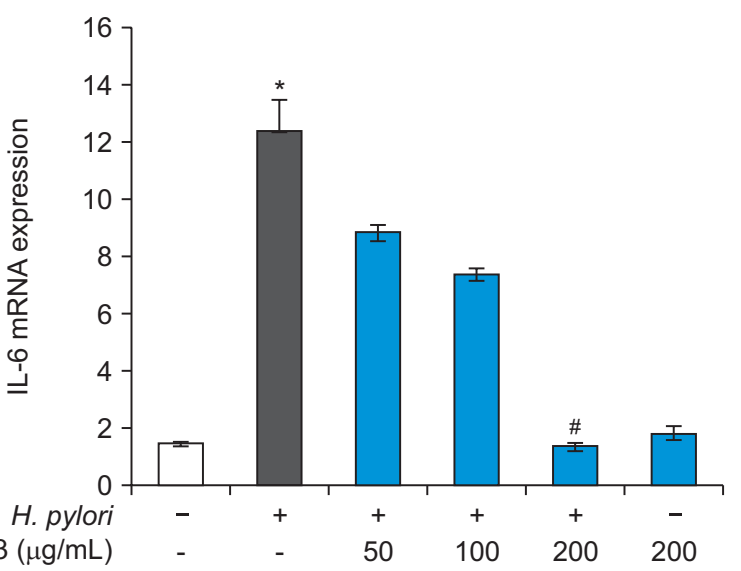

Figure 6. The minimum dose of GGE03 required for exerting anti-inflammatory effects in Helicobacter pylori (H. pylori)-infected AGS cells. AGS cells were pretreated with GGE03 at indicated concentrations for 1 hour, followed by 3.5 hours co-culture with $H$. pylori ( 50 multiplicity of infection [MOI]). The effects of GGE03 on the mRNA expression of IL-8 (A), TNF- $\alpha$ (B), IL-6 (C), and iNOS (D) in AGS cells were determined by quantitative real-time PCR (qRT-PCR). 18S rRNA was used as an internal control for the expression of pro-inflammatory cytokines. Data are the mean \pm SD. Statistical significance was analyzed by ANOVA. IL, interleukin; iNOS, inducible NOS. ${ }^{*} P<0.05$ and ${ }^{* *} P<0.001$ compared to control; ${ }^{*} P<0.05$, ${ }^{\#} P<0.01$, and ${ }^{\# \#} P<0.001$ compared to the H. pylori group. 
ent types of $H$. pylori strains. In this study, we used the 43504 strain. Because the $H$. pylori 43504 strain is positive for CagA and VacA, it is widely used in gastric inflammation studies [28]. In this study, we compared the inhibitory effects of GE and GGE03 on $H$. pylori growth and $H$. pylori-induced inflammation. The growth inhibitory effect of GGE03 was demonstrated with the $H$. pylori 43504 strain whereas GE had an marginal effect. These results suggest that GGE03 has the potential to protect gastric epithelial cells from virulence factors such as CagA and VacA by inhibiting the growth of $H$. pylori.

Next, we investigated that the anti-inflammatory responses using cultured AGS cells infected with $H$. pylori. The transcription factor $\mathrm{NF}-\mathrm{kB}$ regulates the initiation of inflammatory responses induced upon bacterial infection in gastrointestinal cells $[11,15,29]$. GGE03 was shown to reduce the $H$. pylori-induced up-regulation of pro-inflammatory cytokines such as IL-8, TNF- $\alpha$, IL- 6 and IL- $1 \beta$ by inhibiting NF- $\mathrm{kB}$ activation. This is associated with phosphorylation and subsequent ubiquitin-mediated degradation of $I_{\kappa} B \alpha$, leading to increased nuclear translocation of the NF- $\mathrm{B}$ B subunits, such as $\mathrm{p} 65$ and p50 [30]. This induces the expression of NF-kB-related target genes including IL-8, TNF- $\alpha$, IL- 6 and IL-1 $\beta$ [14]. The treatment with GGE03 inhibited the $H$. pylori-induced phosphorylation of $I_{\kappa} B \alpha$ protein and also significantly reduced the phosphorylation of p65. The results showed that the effect of GGE03 was significantly greater than GE in inflammation responses in $\mathrm{H}$. pylori-infected AGS cells. Moreover, the suppression of pro-inflammatory cytokines such as IL-8 and IL$1 \beta$ was significantly different between the GGE03 and GE at an $100 \mu \mathrm{g} / \mathrm{mL}$ concentration.

Production of NO is a feature of gastric epithelial cells as well as genuine immune cells including macrophages upon inflammatory insult [31-33]. In gastric epithelial cells, $H$. pylori produces NO, a ubiquitous free radical synthesized by the enzyme NO synthetase (NOS), via the oxidation of L-arginine [16]. iNOS, one of the NOS isoforms, is regulated at the transcriptional level when cells are challenged by cytokines or pathogens [31]. The previous studies revealed that generation of $\mathrm{NO}$ activates $\mathrm{NF}-\mathrm{kB}$, thereby triggering the inflammation in tumors and other pathological conditions [34,35]. As a consequence of $H$. pylori infection, iNOS expression and NO production were increased in gastric epithelial cells, which accelerates the inflammatory responses, implicated in gastric diseases including gastritis [16]. In this study, we confirmed that GGE03 treatment significantly reduced NO production and expression of iNOS mRNA in $H$. pylori-infected AGS cells. In addition, we revealed that NO production was inhibited by GGE03 to a greater extent than GE. According to the previous studies, $H$. pylori infection provokes the persistent neutrophil infiltration and up-regulation of IFN- $\gamma$ in the gastric mucosa $[19,36]$. In line with this notion, our data showed that the IFN- $\gamma$ expression and MPO activity were increased in $\mathrm{H}$. pylori infection that were reduced by GGE03 treatment.
Finally, we investigated the minimum inhibitory concentration of GGE03 for anti-inflammatory effect on $\mathrm{H}$. pylori infection. Our study revealed that the IL-8, a representative inflammatory cytokine released upon $H$. pylori infection, was significantly reduced at the $50 \mu \mathrm{g} / \mathrm{mL}$ concentration of GGE03. In contrast, TNF- $\alpha$, IL-6 and iNOS were not significantly inhibited at the same concentration. Furthermore, we calculated the human equivalent dose for the effect of GGE03. GGE03 was found to protect against EtOH/HCL-induced rat gastric mucosal injury at concentrations of 100 to $300 \mathrm{mg} / \mathrm{kg}$ [5]. Based on our findings and others, we estimate that an amount of 400 to $1,000 \mathrm{mg} /$ day GGE03 is required for a $60 \mathrm{~kg}$ subject as calculated by the recommended body surface area normalization method [37]. However, in order to determine the exact concentration of the anti-inflammation effect of GGE03 against $H$. pylori infection, an in vivo studies need to be conducted.

To sum up, GGE03 suppresses the expression of pro-inflammatory cytokines, NO production and MPO activity in $H$. pylori-infected gastric epithelial cells. GGE03 appears to have more potent anti-inflammation effects compared to GE. Collectively, it is possible that GGE03 might serve as a preventive formula for $H$. pylori-associated gastritis.

\section{ACKNOWLEDGMENTS}

This work was supported by the GRRC program of Gyeonggi province (GRRC-201900830002-CHA2019-B01, Production of physiologically active substances), Republic of Korea.

\section{CONFLICTS OF INTEREST}

No potential conflicts of interest were disclosed.

\section{ORCID}

Moon-Young Song, https://orcid.org/0000-0001-9311-5146

Da-Young Lee, https://orcid.org/0000-0001-7655-6740

Sang-Yong Park, https://orcid.org/0000-0003-0609-7897

Seul-A Seo, https://orcid.org/0000-0002-9640-9729

Jeong-Seung Hwang, https://orcid.org/0000-0003-0505-2312

Soo-Hyeon Heo, https://orcid.org/0000-0002-0105-0355

Eun-Hee Kim, https://orcid.org/0000-0002-8523-0440

\section{REFERENCES}

1. Terry R, Posadzki P, Watson LK, Ernst E. The use of ginger (Zingiber officinale) for the treatment of pain: a systematic review of clinical trials. Pain Med 2011;12:1808-18.

2. Chen CX, Barrett B, Kwekkeboom KL. Efficacy of oral ginger (Zingiber officinale) for dysmenorrhea: a systematic review and meta-analysis. Evid Based Complement Alternat Med 2016; 2016:6295737.

3. Mashhadi NS, Ghiasvand R, Askari G, Hariri M, Darvishi L, 
Mofid MR. Anti-oxidative and anti-inflammatory effects of ginger in health and physical activity: review of current evidence. Int $\mathrm{J}$ Prev Med 2013;4(Suppl 1):S36-42.

4. Park SH, Jung SJ, Choi EK, Ha KC, Baek HI, Park YK, et al. The effects of steamed ginger ethanolic extract on weight and body fat loss: a randomized, double-blind, placebo-controlled clinical trial. Food Sci Biotechnol 2019;29:265-73.

5. Shin JK, Park JH, Kim KS, Kang TH, Kim HS. Antiulcer activity of steamed ginger extract against ethanol/ $\mathrm{HCl}$-induced gastric mucosal injury in rats. Molecules 2020;25:4663.

6. Nam YH, Hong BN, Rodriguez I, Park MS, Jeong SY, Lee YG, et al. Steamed ginger may enhance insulin secretion through $\mathrm{K}_{\text {ATP }}$ channel closure in pancreatic $\beta$-cells potentially by increasing 1-dehydro-6-gingerdione content. Nutrients 2020;12:324.

7. Cover TL, Blaser MJ. Helicobacter pylori in health and disease. Gastroenterology 2009;136:1863-73.

8. Suerbaum S, Michetti P. Helicobacter pylori infection. N Engl J Med 2002;347:1175-86.

9. Münzenmaier A, Lange C, Glocker E, Covacci A, Moran A, Bereswill S, et al. A secreted/shed product of Helicobacter pylori activates transcription factor nuclear factor-кB. J Immunol 1997; 159:6140-7.

10. Lamb A, Chen LF. Role of the Helicobacter pylori-induced inflammatory response in the development of gastric cancer. J Cell Biochem 2013;114:491-7.

11. Lamb A, Yang XD, Tsang YH, Li JD, Higashi H, Hatakeyama $\mathrm{M}$, et al. Helicobacter pylori CagA activates NF- $\mathrm{KB}$ by targeting TAK1 for TRAF6-mediated Lys 63 ubiquitination. EMBO Rep 2009;10:1242-9.

12. Song MY, Lee DY, Kim EH. Anti-inflammatory and anti-oxidative effect of Korean propolis on Helicobacter pylori-induced gastric damage in vitro. J Microbiol 2020;58:878-85.

13. Wilson M, Seymour R, Henderson B. Bacterial perturbation of cytokine networks. Infect Immun 1998;66:2401-9.

14. Sharma SA, Tummuru MK, Blaser MJ, Kerr LD. Activation of IL-8 gene expression by Helicobacter pylori is regulated by transcription factor nuclear factor- $\mathrm{\kappa B}$ in gastric epithelial cells. $J$ Immunol 1998;160:2401-7.

15. Cho K, Lee HG, Piao JY, Kim SJ, Na HK, Surh YJ. Protective effects of silibinin on Helicobacter pylori-induced gastritis: NF-kB and STAT3 as potential targets. J Cancer Prev 2021;26:118-27.

16. Gobert AP, Wilson KT. The immune battle against Helicobacter pylori infection: no offense. Trends Microbiol 2016;24:366-76.

17. Faith $M$, Sukumaran A, Pulimood AB, Jacob M. How reliable an indicator of inflammation is myeloperoxidase activity? Clin Chim Acta 2008;396:23-5.

18. Han H, Lim JW, Kim H. Astaxanthin inhibits Helicobacter pyloriinduced inflammatory and oncogenic responses in gastric mucosal tissues of mice. J Cancer Prev 2020;25:244-51.

19. Abdollahi H, Shams S, Zahedi MJ, Darvish Moghadam S, Hayatbakhsh MM, Jafarzadeh A. IL-10, TNF- $\alpha$ and IFN- $\gamma$ levels in serum and stomach mucosa of Helicobacter pylori-infected patients. Iran J Allergy Asthma Immunol 2011;10:267-71.

20. Fan XJ, Chua A, Shahi CN, McDevitt J, Keeling PW, Kelleher D.
Gastric T lymphocyte responses to Helicobacter pylori in patients with H pylori colonisation. Gut 1994;35:1379-84.

21. Zamani M, Ebrahimtabar F, Zamani V, Miller WH, Alizadeh-Navaei $\mathrm{R}$, Shokri-Shirvani J, et al. Systematic review with meta-analysis: the worldwide prevalence of Helicobacter pylori infection. Aliment Pharmacol Ther 2018;47:868-76.

22. Ansari S, Yamaoka Y. Helicobacter pylori virulence factor cytotoxin-associated gene A (CagA)-mediated gastric pathogenicity. Int J Mol Sci 2020;21:7430.

23. Selbach M, Moese S, Hauck CR, Meyer TF, Backert S. Src is the kinase of the Helicobacter pylori CagA protein in vitro and in vivo. J Biol Chem 2002;277:6775-8.

24. Atherton JC. The pathogenesis of Helicobacter pylori-induced gastro-duodenal diseases. Annu Rev Pathol 2006;1:63-96.

25. Zhang YX, Zhou LY, Song ZQ, Zhang JZ, He LH, Ding Y. Primary antibiotic resistance of Helicobacter pylori strains isolated from patients with dyspeptic symptoms in Beijing: a prospective serial study. World J Gastroenterol 2015;21:2786-92.

26. Ghotaslou R, Leylabadlo HE, AsI YM. Prevalence of antibiotic resistance in Helicobacter pylori: a recent literature review. World J Methodol 2015;5:164-74.

27. Israel DA, Salama N, Arnold CN, Moss SF, Ando T, Wirth HP, et al. Helicobacter pylori strain-specific differences in genetic content, identified by microarray, influence host inflammatory responses. J Clin Invest 2001;107:611-20.

28. Honda S, Fujioka T, Tokieda M, Gotoh T, Nishizono A, Nasu M. Gastric ulcer, atrophic gastritis, and intestinal metaplasia caused by Helicobacter pylori infection in Mongolian gerbils. Scand J Gastroenterol 1998;33:454-60.

29. Kim JG, Lee SJ, Kagnoff MF. Nod1 is an essential signal transducer in intestinal epithelial cells infected with bacteria that avoid recognition by toll-like receptors. Infect Immun 2004;72:1487-95.

30. Tak PP, Firestein GS. NF-kB: a key role in inflammatory diseases. J Clin Invest 2001;107:7-11.

31. Bogdan C. Nitric oxide and the immune response. Nat Immunol 2001;2:907-16.

32. Martín MC, Martinez A, Mendoza JL, Taxonera C, Díaz-Rubio $M$, Fernández-Arquero $M$, et al. Influence of the inducible nitric oxide synthase gene (NOS2A) on inflammatory bowel disease susceptibility. Immunogenetics 2007;59:833-7.

33. MacMicking J, Xie QW, Nathan C. Nitric oxide and macrophage function. Annu Rev Immunol 1997;15:323-50.

34. Kleinert H, Wallerath T, Fritz G, Ihrig-Biedert I, RodriguezPascual F, Geller DA, et al. Cytokine induction of NO synthase II in human DLD-1 cells: roles of the JAK-STAT, AP-1 and NF-kBsignaling pathways. Br J Pharmacol 1998;125:193-201.

35. Ganster RW, Taylor BS, Shao L, Geller DA. Complex regulation of human inducible nitric oxide synthase gene transcription by Stat 1 and NF-кB. Proc Natl Acad Sci USA 2001;98:8638-43.

36. Robinson $\mathrm{K}$, Argent $\mathrm{RH}$, Atherton JC. The inflammatory and immune response to Helicobacter pylori infection. Best Pract Res Clin Gastroenterol 2007;21:237-59.

37. Nair AB, Jacob S. A simple practice guide for dose conversion between animals and human. J Basic Clin Pharm 2016;7:27-31. 